\title{
Hypothesis: Control of hepatic utilization of alanine by membrane transport or by cellular metabolism?
}

\author{
Review
}

\author{
Halvor N. Christensen \\ Department of Biological Chemistry, \\ The University of Michigan Medical School, \\ Ann Arbor, Michigan 48109, U.S.A.
}

The rate of alanine transport into the liver limits its utilization even under the high alanine load resulting from a $90 \%$ casein diet, given that the rat has been adapted to that diet. A coordinated acceleration of alanine catabolism allows transport to remain ratelimiting, which in turn allows the adaptive regulation of transport to remain effective at high alanine loads. Accelerated degradation of alanine may change the hepatic amino acid content in a way that derepresses the activity of the alanine carrier system(s).

\section{Introduction}

Alanine is the main glucogenic amino acid flowing to the liver, and a major source of pyruvate for the hepatocyte (Fig. 1). Transport across the plasma membrane is the first step in alanine metabolism. This transport occurs largely by two $\mathrm{Na}^{+}$-dependent systems: one, System $A$, is subject to adaptive regulation by the hepatocyte itself and to hormonal regulation (1). $\mathrm{Na}^{+}$-independent transport of alanine is ordinarily small, although not negligible.

The catabolism of alanine might be accelerated by its intensified transport, but perhaps also by induction of alanine aminotransferase (2) and by changes in the rate of subsequent disposition of pyruvate. What then is the relative part played by modulation of enzyme activity compared with changes in the rate of membrane transport, in determining the alanine flow to the liver? This question has obvious counterparts in the regulation of other interorgan flows of the amino acids $(1,3)$, e.g. in the flow of glutamine from muscle or to the kidney. So special is the case of alanine flow to the liver, however, that the answers to the above question may well prove not to apply closely to flows of other amino acids.

Three recent in vitro studies on this question have concluded that transport across the plasma membrane will generally be rate-limiting to hepatic alanine utilization (4-6). One of these indicated that at external alanine levels above $1 \mathrm{mM}$, this would no longer be the case (5). The alanine content of hepatocytes had previously been shown by Sips et al. (4) to remain low, about $1 \mathrm{mM}$, as the external alanine 


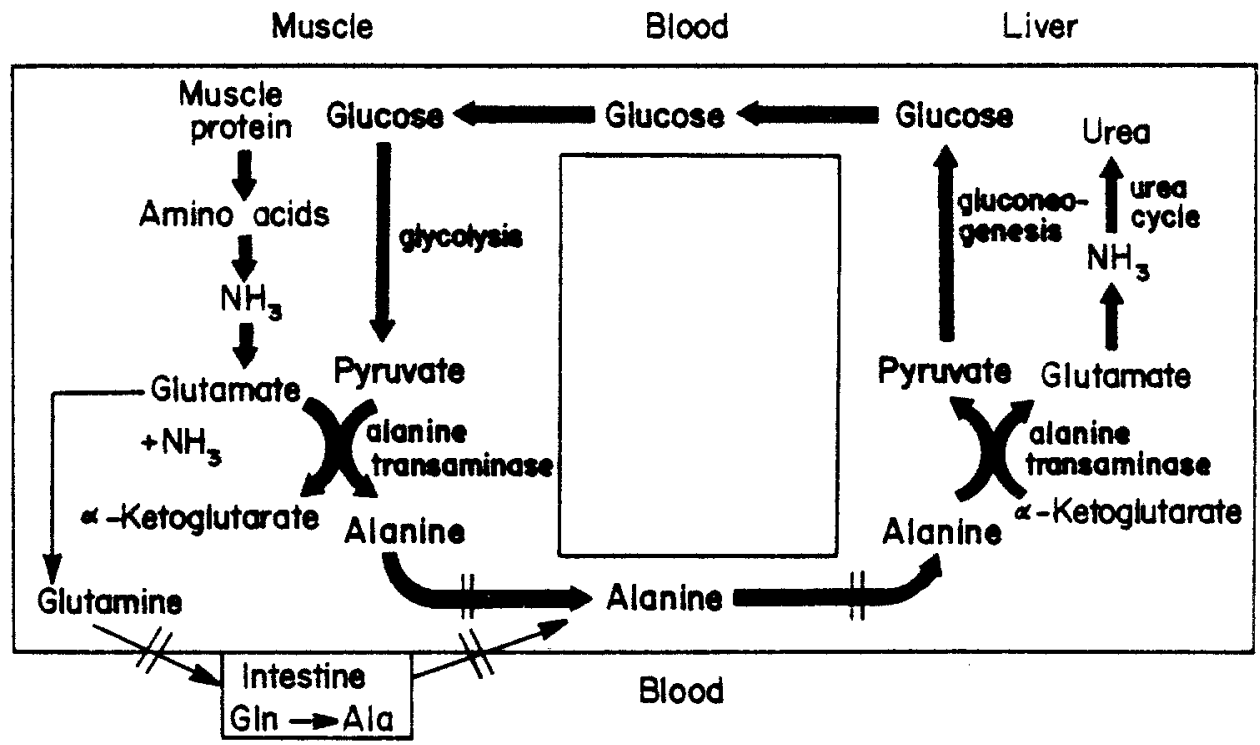

Fig. 1. The glucose-alanine cycle, adapted from Lehninger, Principles of Biochemistry, 1982, to reflect the participation of glutamine in the flow, and to represent with the double vertical lines some points at which plasma-membrane transport of amino acids intervenes.

was increased and alanine utilization accelerated. A more recent study from the same laboratory found the hepatic alanine concentration still below that of the external medium at $6 \mathrm{mM}$, which was the highest level tested (6).

\section{A Nutritional Study}

A new study $(7,8)$ calls attention to the desirability that such studies be made also in vivo, and with test animals adapted to high or low protein intake. Under these conditions the rate-limiting step might change with nutritional status, as well as with the alanine load imposed. In vivo experiments provide, I urge, an added advantage, arising out of the complex way in which amino acids reach the liver. Thus the hepatocytes first encountered by the portal flow undoubtedly have a richer and qualitatively different amino acid pattern than do the cells exposed later in the sequence. This situation contrasts with experiments using suspended cells, where a single alanine concentration is instead selected for each test. In the in vivo studies $(7,8)$, rats were fed diets containing 5, 13, 50, or $90 \%$ casein for 21 days to secure adaptation to the respective protein intakes. Wheat starch served to bring the sum of the two main dietary constitutents to $90 \%$. Corn oil plus $5 \%$ salt mixture and $1 \%$ vitamins completed the diet. The rats on $90 \%$ casein therefore had to meet most of their energy needs from this protein. Male Wistar rats of 180 to $200 \mathrm{~g}$ were provided access to one of these rations during the first $8 \mathrm{~h}$ of 
darkness in a day split $12 \mathrm{~h}: 12 \mathrm{~h}$ between light and dark. Food consumption and weight gain on these programs were recorded in an earlier study (8).

Some of the rats to be designated as fed were then sacrificed for analysis 4 to $5 \mathrm{~h}$ after the beginning of food intake. This choice means that the high-casein rats, for example, were under two stimuli: the adaptive effects of the regime for the previous 21 days; and the immediate acute effects of a corresponding load of alanine and other amino acids at the time of sampling. Some of the rats receiving $13 \%$ casein were instead sampled $48 \mathrm{~h}$ after food removal; these rats were designated as starved. The rats were anesthetized with sodium pentobarbital, and held at $37^{\circ}$ for laparotomy. Blood samples were drawn slowly and successively from two selected vessels, either from the portal vein and then from the aorta, or from the hepatic vein and then from the portal vein. A constant flow of p-aminohippurate into a mesenteric vein had been in the meanwhile maintained. This indicator served then by dilution to measure the portal, or the hepatic, blood flow. From these data the afferent concentration of alanine for the liver was calculated. This calculation slightly lowered the alanine load that might have been calculated from analyses of the portal alanine level alone. Hepatic and vascular alanine, hepatic glutamate, pyl.uvate, 2-ketoglutarate, and alanine aminotransferase were measured by standard enzymologic methods.

In the fed rats, arterial concentrations of alanine were hardly affected by the roughly 18 -fold change in protein intake brought about by shift from $5 \%$ to $90 \%$ casein, although the alanine levels in the portal plasma tripled over this range (Fig. 2). Hence the extra alanine level provided by the portal blood was largely cleared by the

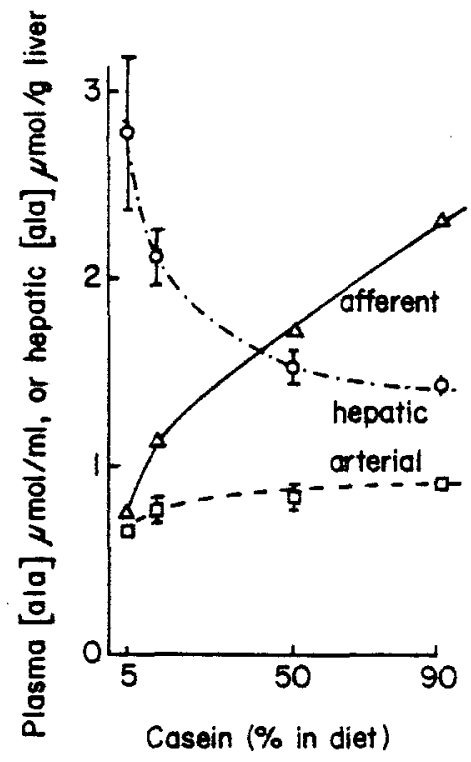

Fig. 2. Concentrations of alanine in arterial and hepatic-afferent plasma and in the liver of rats fed at various protein levels in their diets. (Adapted from Fafournoux et al., reference 7 ). 
liver. In contrast arterial leucine rose nearly 5-fold (9), so obviously some amino acids are not extracted by the liver as extensively as alanine is. These results point to a possible flaw in earlier work where too narrow a range of alanine concentrations was selected to demonstrate that alanine transport in vitro is rate-limiting. The range regularly encountered in arterial blood is about 0.2 to $0.5 \mathrm{mM}$ alanine, one that probably does not represent the full range of physiological interest. In starved rats afferent plasma and hepatic alanine levels fell. A major factor in the stability of the arterial alanine level across the wide range of casein intake was the accelerated hepatic utilization of alanine, which almost kept pace with any increased supply. After the adaptation to $90 \%$ casein, this utilization was increased 5-fold. The fractional extraction of alanine from the portal blood rose from less than $20 \%$ at $5 \%$ casein intake to over $70 \%$ on the $90 \%$ casein diet.

Hepatic alanine fell surprisingly to about one half as a consequence of the high-protein regime (Fig. 2). The concomitant rise in portal alanine and the fall in hepatic alanine thus gave rise by changes both in the numerator and the denominator to gradients more favorable to hepatic uptake. In the starved rats in contrast alanine extraction was limited to subnormal amounts by the low levels in the portal blood.

Alanine aminotransferase activity, which is present in excess of the apparent normal needs, was measured in the fed rats and found to be quadrupled after accommodation to the high protein intake. Nevertheless the hepatic mass action ratio of the four reactants of this enzyme action, which measures the spontaneity of the reaction, was scarcely changed from about unity despite the large increase in alanine utilization. Hence subsequent metabolism of the products must have been correspondingly accelerated. Alanine catabolism not only kept up with the alanine supply; it ran ahead of it under the stimulus presumably provided by adaptation to the high protein intake. These results provide us with much the same answer as Groen et al. reported (6): alanine transport remains rate-limiting to its metabolism up to the concentration of about $2 \mathrm{mM}$, the level obtained in the afferent blood plasma after 4 to $5 \mathrm{~h}$ of eating the $90 \%$ casein diet. This alanine load should represent a sufficient test of the question posed in the title of this article.

\section{Adaptive Regulation of Amino Acid Transport Would Be Futile Unless Transport Is Rate-limiting to Generation or Consumption of the Amino Acid}

A new finding from our laboratory intensifies our interest in an answer to the title question. The answer determines, as far as we can understand, whether adaptive regulation (10) succeeds or not. By adaptive regulation we mean the stimulation of amino acid transport into cells seen during amino acid deprivation and the repression of that transport by excesses of certain amino acids. Using an experimental hepatoma cell line, we have found that adaptive regulation modulates roughly equally the inward and the outward fluxes by System A (11). Therefore adaptive regulation will apparently be futile whenever the two fluxes are permitted to come to equilibrium. In other words, transport must remain rate-limiting for adaptive control to serve a useful purpose. 


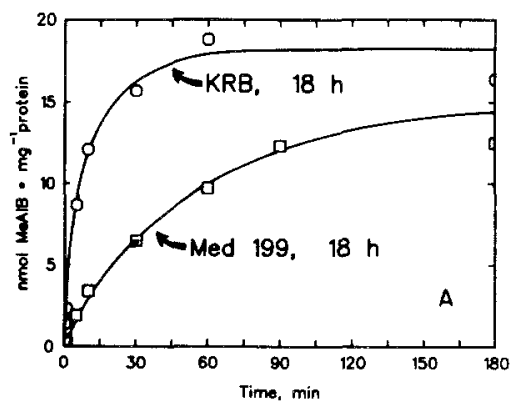

Fig. 3. Time course of the uptake of $\left[{ }^{14} \mathrm{C}\right] 2-$ (methylamino)isobutyric acid by hepatoma cells from a $0.2 \mathrm{mM}$ medium. The cells had first been held for $18 \mathrm{~h}$ either in amino-acid-free Krebs-Ringer bicarbonate medium, or (lower curve) in the standard amino-acid-rich culture medium 199. Subsequently the cells were treated for $3 \mathrm{~h}$ with the modified Earle's balanced salt solution containing $20 \mu \mathrm{M}$ cycloheximide to 'freeze' the cells at the established regulative state. The curves compare the subsequent approaches to steady-state distribution of the test amino acid.

Fig. 3 illustrates the approach, apparently to somewhat the same equilibrium distribution, for 2-(methylamino) isobutyric acid (MeAIB) in cells treated with amino-acid-free and with amino-acid-rich media, although this equilibrium still appears several hours away. For an amino acid that undergoes extensive hepatic metabolism, an equality of the two transmembrane fluxes (and hence a zero balance between the adaptive effects on influx and efflux) is less likely to be attained, and is not proved by attainment of a steady state. We showed years ago (12) that amino acids suffer competition in the sustaining of concentration gradients between liver and plasma. The more recent results of Bloxam (13) showed that starvation enhances (a) the hepatic concentrating ability for most of the amino acids flowing toward the liver, and (b) the outward flow of those amino acids which are released by the liver. Threonine transport into and out of the liver was found rate-limiting to its catabolism (14), allowing an adaptive regulation to conserve this nutrient.

Fafournoux et al. (7) were, however, investigating principally a regulation that seemed to go beyond adaptive regulation, namely a stimulation rather than a repression by excess transport substrate, which is just what is needed to allow the organism to succeed in eliminating dietary amino acid excesses. Yet the hepatic alanine was actually lowered (Fig. 2) by adaptation to the high casein intake. White and I show that it is probably the intracellular and not the extracellular or membrane levels of the effective amino acids that determine adaptive regulation (11). The cellular accumulation of $\mathrm{Na}^{+}$, produced by increasing levels of ouabain, serves to reversibly prevent (a) rises in the internal MeAIB level and (b) the usual transportrepressive action of external NeAIB. Otherwise ouabain was without 
effect on adaptive regulation. Hence an accelerated hepatic conversion of repressive amino acids (e.g. alanine, glutamine) to substances not repressive to neutral amino acid transport (e.g. pyruvate, glutamate) might explain the transport adaptation seen by Fafournoux et al. on high casein intake. Could a modification of catabolism then prove to be the primary change? Neutral amino acids other than alanine may have been lowered in the liver by the high casein intake. A complicating factor is the demonstration that the administration of glutamate to the rat stimulates the hepatic accumulation of other amino acids into liver and muscle (12), an effect apparently still largely unexplained.

In any case a valuable paradox emerges from the work of Fafournoux et al.: To retain optimal control at the level of membrane transport, metabolic consumption of alanine must be accelerated sufficiently with a rising amino acid load for transport to remain rate-limiting. Otherwise adaptive regulation will soon cease to serve. In short, regulation of hepatic alanine utilization occurs at the level of transport, but metabolism must also be modulated if that regulation is to be maintained over a reasonable range of loads.

\section{High Casein Intake and Hepatocyte Accumulation of Alanine in Vitro}

Fafournoux et al. proceed to show that adaptation of rats to high casein intake had indeed stimulated hepatic alanine transport in isolated hepatocytes. The present work does not seem to improve on their earlier demonstration (8) that System A is the transport system principally stimulated by the high-casein diet. This system is surprisingly called (7) a 'high-affinity system', although half-saturation occurred at about $4 \mathrm{mM}$. Syster, A has previously proved especially responsive to regulation. The new results uncover a remarkable accumulative capacity for extra alanine, demonstrable by bringing the external alanine level to about $6 \mathrm{mM}$ with pure alanine, all in the presence of aminoxyacetate (Fig. 4). This demonstration includes an upward concavity of the curves relating hepatocyte alanine accumulation to the external load. This form is a dramatic reversal from the hyperbolic curves usually seen for cells and tissues, as saturation of their capacity for amino acid accumulation is approached. For the $90 \%$-casein-fed group the partition coefficients of intracellular to extracellular alanine rose from a value of two to a value of about five when the external alanine was doubled in vitro. Since a separate plot showed influx rising less than linearly with rising external alanine concentration, the increased partition coefficients at high alanine loads appear to arise from decreased efflux. The accumulation from $4 \mathrm{mM}$ external alanine level had more than doubled after adaptation to the $90 \%$ casein diet.

\section{Accumulative Capacity of Liver for Amino Acids in Vivo}

Even stronger responses were obtained when alanine was injected in order to raise the portal plasma alanine to a similar level (7). (In this case aminoxyacetate presumably was not used to check alanine catabolism.) These in vivo results showed a remarkable and paradoxic 


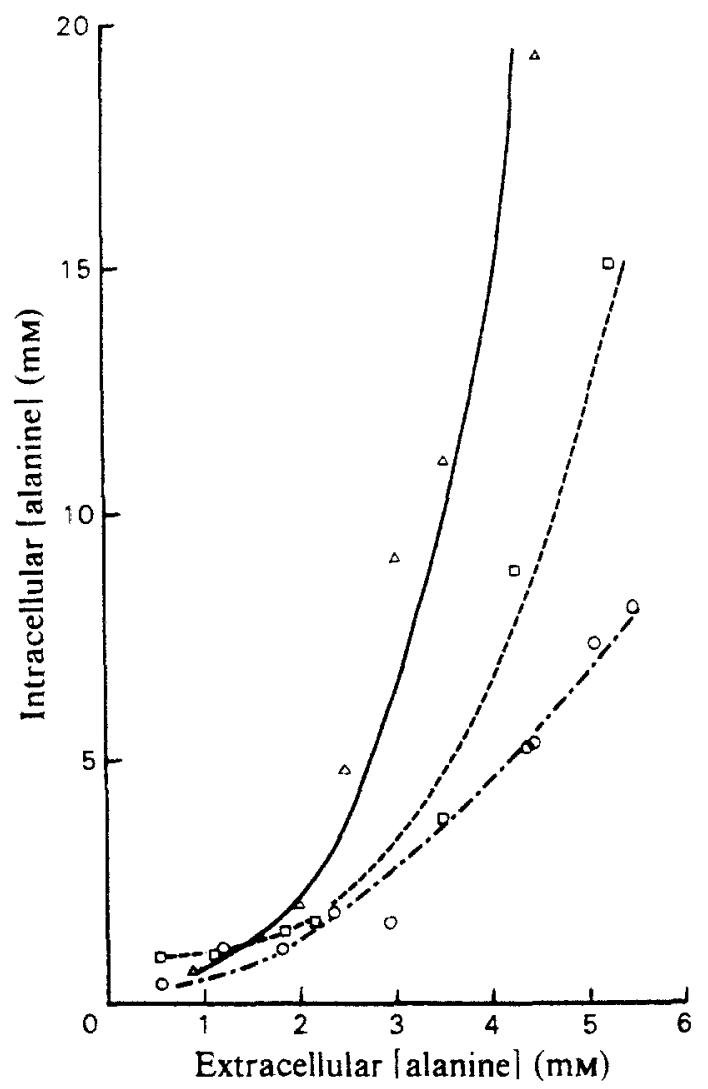

Fig. 4. Effects of external concentrations on intracellular alanine attained in vitro during 10 min. Alanine levels ranged from 0.6 to $6 \mathrm{mM}$, from fed rats maintained on $13 \%(\square)$ or $90 \%(\Delta)$ casein diets, or from rats of the first group fasted $48 \mathrm{~h}$. From Fafournoux et al. (reference 7), with permission.

capacity of the liver to accumulate alanine supplied as alanine, over the accumulation seen when alanine was fed in the form of casein.

In their classical study seven decades ago, Van Slyke and Meyer (15) showed that the liver has a very large capacity for accumulating infused amino acids. They infused into each of two dogs during 30 min $32 \mathrm{mmol}$ of amino acids per $\mathrm{kg}$ of body weight in the form of acid-hydrolyzed casein. Blood and tissues were removed for analysis half an hour later. At the amino acid levels of nearly $30 \mathrm{mM}$ that were obtained in femoral venous blood, the hepatic levels had increased by about $44 \mathrm{mmol}$ per $\mathrm{kg}$ of liver, corresponding to about 81 $\mathrm{mM}$ added amino acid in the cellular water, which is nearly three times the increment seen for the plasma. Perhaps I may say that this and the attendant discoveries of Van Slyke and Meyer were so premature that their significance has not yet been widely appreciated. 
Neither that $81 \mathrm{mM}$ level, nor the incremental alanine accumulation of perhaps $24 \mathrm{mM}$ produced by Fafournoux et al. by alanine infusion into the portal vein of the rat, necessarily represents approaching saturation of the hepatic capacity for amino acid accumulation. Had Van Slyke infused more casein hydrolysate, or Fafournoux more alanine, still higher accumulations would undoubtedly have been attained. Nevertheless some limits must apply. Tests with the Ehrlich cell have repeatedly shown competitive interactions among the amino acids for accumulation (3). The hepatic accumulations Fafournoux et al. obtained on portal infusions of alanine cry for investigation of the competitive interactions and regulatory phenomena among amino acids at high levels. The immediately prior feeding with high casein may have generated extra capacity for hepatic accumulation of alanine by exchange with other intracellular amino acids.

I believe, however, that for examining my title question the experiments of Fafournoux et al. with pure alanine involve doses in excess of the physiological alanine load. The rats on the casein diet showed hepatic-afferent alanine levels up to $2.3 \mathrm{mM}$, which led only to declines, not increases, of hepatic alanine levels from those seen with lower alanine loads. Alanine flow from the intestine to the liver will include not only the $3.2 \%$ alanine in casein, but also substantial quantities of alanine formed in the intestinal tissue from other amino acids, as illustrated in Fig. 1. I suggest then that alanine loads of up to a full $2 \mathrm{mM}$ concentration cover adequately the physiological range. The paradoxical results obtained with pure alanine, which do not refute the answer provided by Fig. 2, introduce a different problem, although a provocative one.

\section{A Test of Prior Fasting of Rats on Hepatocyte Uptake of Alanine Hepatocytes}

A different study by Kristensen et al. (16) considered especially the effect of a $48-\mathrm{h}$ fast in rats. The alanine gradient maintained by the hepatocytes treated in vitro with aminoxyacetate was about doubled by the fast. This increase arose from 2- to 3-fold increase of mediated influx, accompanied by a $50 \%$ increase in efflux, not completely characterized as to route. The relation between these changes is consistent with equal effects of adaptive regulation on both fluxes, given that the mediated fluxes under stimulation would by no means be equal. That is, a disproportionate part of the efflux undoubtedly occurs by a route not subject to adaptive regulation.

\section{Summary}

Fed rats adapted to a high casein intake maintain elevated rates of hepatic extraction of alanine, with the result that nearly all incremental alanine is extracted by the liver. Much of this alanine is catabolized. In contrast, a high intake of alanine as such leads to a high hepatic alanine content. For transport to remain rate-limiting, as it does, at higher loads, the catabolism of alanine must increase. This result correlates with the finding that adaptive regulation of transport System A modifies simultaneously both influx and efflux. The regulation accordingly becomes futile if the transport process were 
allowed to reach equilibrium. Modulation of amino acid catabolism, by changing the level of repressive amino acids, should influence the response of the transport system. These changes might even allow derepression of hepatic amino acid transport during adaptation to high protein intake. Studies with isolated hepatocytes show that prior fasting of rats also intensified alanine uptake, efflux also being stimulated although rather less than influx.

\section{Acknowledgement}

Work originating from this laboratory has been supported by a grant (HDO1233) from the Institute for Child Health and Human Development, National Institutes of Health, U.S. Public Health Service.

\section{References}

1. Christensen HN (1982) Physiol. Rev. 62, 1193-1233.

2. Kaplan JH \& Pitot HC (1970) in Mammalian Protein Metabolism (Munro HN, ed), Academic Press, London \& New York, pp 387-443.

3. Christensen HN (1955) in Amino Acid Metabolism (McElroy WD \& Glass B, eds), The Johns Hopkins Press, Baltimore, pp 63-106.

4. Sips HJ, Groen AK \& Tager JM (1980) FEBS Lett. 119, 271-274.

5. McGivan JD, Ramse11 JC \& Lacey JH (1981) Biochim. Biophys. Acta 644, 295-304.

6. Groen A, Sips HJ, Vervoorn RC \& Tager JM (1982) Eur. J. Biochem. 122, 87-93.

7. Fafournoux P, Rémésy C \& Demigné C (1983) Biochem. J. 210, 645-652.

8. Fafournoux P, Rémésy C \& Demigné C (1982) Biochem. J. 206, 13-18.

9. Rémésy C \& Demigné C (1982) J. Nutr. 112, 60-69.

10. Guidotti GG, Borghetti AF \& Gazzola GC (1978) Biochim. Biophys. Acta 515, 329-366.

11. White MF \& Christensen HN (1983) J. Biol. Chem. 258, 8028-8038.

12. Christensen HN, Streicher JA \& Elbinger RL (1948) J. Biol. Chem. 172, 515-524.

13. Bloxam DL (1972) Brit. J. Nutr. 270, 233-247.

14. Bloxam DL (1975) Am. J. Physiol. 229, 1718-1723.

15. Van Slykes DD \& Meyer GM (1913-1914) Bio1. Chem. 16, 197-212.

16. Kristensen L $\varnothing$, Sestoft L \& Folke M (1983) Am. J. Physiol. 244, G491-G500. 